\title{
Tinnitus - a manifestation of neurodegenerative diseases
}

\author{
Boris Borisov', Mario Milkov², Miroslav Stoykov³, Elena Dimova ${ }^{4}$ \\ 'Head of Training sector "Dental technician", Medical college, Medical University - Varna \\ ${ }^{2}$ Head of Department of Dental materials science and propaedeutics of prosthetic dental medicine, \\ Faculty of Dental medicine, Medical University - Varna \\ ${ }^{3}$ Assistant professor, Department of Dental materials science and propaedeutics of prosthetic dental medicine, \\ Faculty of Dental medicine, Medical University - Varna \\ ${ }^{4}$ Assistant professor, Department of Pediatric Dental Medicine, Faculty of Dental medicine, \\ Medical University - Varna
}

\begin{abstract}
\section{Background}

Tinnitus is a widespread hearing impairment that can be characterized by the perception of sound, such as tone or noise, in the absence of an appropriate external sound source. The cause of tinnitus and mechanism are unclear. The prevalence of tinnitus is higher in people over 60 years of age. Tinnitus is usually a manifestation of a hearing impairment. Symptoms can be acute or chronic. In most cases, tinnitus manifests as an idiopathic condition. In the clinical practice, common factors that affect the psychological and emotional well-being of people with tinnitus are: fear, stress, anxiety and depression, which in turn can cause insomnia, impaired concentration and cognitive dysfunction. Tinnitus is a heterogeneous disorder in terms of its etiology and clinic. Tinnitus is associated with hearing loss, as both symptoms often occur together, though in many clinical cases, people with hearing loss do not develop tinnitus.
\end{abstract}

\section{Methods}

We present a clinical case of a patient diagnosed with a posturographic system, along with a full otoneurological and hearing examinations. Methods of videonystagmography, video head impulse test, subjective visual vertical, audiometry, tympanometry, otoacoustic emissions test, automated auditory brainstem response were used. We provided a dental check-up and an occlusion analysis. Patient filled out questionnaires and signed a written informed consent. The aim of the team was to analyze the manifestation of tinnitus as a precursor of a neurodegenerative disease.

\section{Results and discussion}

From the literature review done chronic tinnitus is associated with anatomical brain abnormalities, including decreased cortical gray matter and decreased white matter integrity. There is also a high prevalence of cognitive impairment reported among patients with tinnitus from a clinical point of view. Tinnitus is an independent risk factor for subsequent neurodegenerative disease. There are a number of neurological conditions that can be encountered in the otorhinolaryngological and dental practice. It is important for the dentist to enrich own knowledge of the underlying neurological conditions, as they may affect the provision of dental treatment. Our findings from the examinations executed are consistent with previous studies, which show that diabetes and head injuries can be risk factors for $\mathrm{AD}$ and neurodegeneration.

\section{Conclusions}

Further research is needed to re-confirm the conclusions of our research and to investigate the pathophysiology of the relationship between tinnitus and the Alzheimer's disease and tinnitus and the Parkinson's disease in more depth.

Key words: tinnitus, hearing loss, ringing, neurodegenerative, Parkinson's disease, Alzheimer's disease

\section{Background}

Tinnitus, also called "ringing in the ears" or phantom sound perception, is a widespread hearing impairment that can be characterized by the perception of sound, such as tone or noise, in the absence of an appropriate external sound source [1]. According to studies, the overall prevalence of tinnitus in the elderly population is about $10 \%$ [2]. The prevalence of tinnitus is higher (12-18\%) in people over 60 years of age [3]. Tinnitus is usually a manifestation of a hearing impairment [4]. Symptoms can be acute (beginning in the last 3 months) or chronic (usually lasting more than 12 months). In rare cases, an objective cause of the ringing that can be treated, is identified. In most cases, however, tinnitus is a subjective symptom and manifests as an idiopathic condition, the exact mechanism of which remains unknown. In the clinical practice, common factors that affect the psychological and emotional well-being of people with tinnitus are: fear, stress, anxiety and depression, which in turn can cause insomnia, impaired concentration and cognitive dysfunction [5]. Various brain functions, such as learning, emotion, memory, concentration and behavior, are related to the neural connections of sensory processing of tinnitus [6].

Tinnitus is a heterogeneous disorder in terms of its etiology and clinic. In many clinical cases, tinnitus is associated with hearing loss, as both symptoms often occur together. Approximately $90 \%$ of people with chronic tinnitus have some form of a hearing loss [7]. In addition, the acoustic characteristics of the sensation of tinnitus correspond to 
the area of hearing loss: high-pitched tinnitus is usually accompanied by high-frequency hearing loss [8]. About $10 \%$ of people with tinnitus have normal hearing thresholds $(\leq 20 \mathrm{~dB}$ hearing level at frequencies from 0.25 to $8 \mathrm{kHz}$ ) in a standard clinical audiometric examination [9]. Many people with hearing loss do not develop tinnitus.

Table 1 shows the four different types of tinnitus. (Table 1)

Table 2 shows some of the subtypes of tinnitus. (Table 2)

Usually the cause of tinnitus is unclear. Figure 1 shows some of the main causes of tinnitus. (Fig. 1)

\section{Methods}

We present a clinical case of a patient (male, aged 55), diagnosed, using the newly implemented system posturographic system, along with a full otoneurological and hearing examination. Methods of videonystagmography (VNG), video head impulse test (vHIT), subjective visual vertical (SVV), audiometry, tympanometry, otoacoustic emissions test (OAE-test), automated auditory brainstem response (SERA) were used. We provided a dental check-up and an occlusion analysis. Patient filled out questionnaires (dental and vestibular questions included) and signed a written informed consent. The aim of the team was to analyze the manifestation of tinnitus as a precursor of a neurodegenerative disease (in the specific clinical case - of a newly diagnosed Parkinson disease case).

\section{Results and discussion}

In some patients diagnosed with a central-type of tinnitus, the evidence points to the clinical picture - a progressive loss of a neuronal function - as the first sign of a neurodegenerative disease of the central nervous system [10]. Neurodegenerative diseases are, for instance, Alzheimer's disease (AD) and Parkinson's disease (PD). They are characterized by a gradual progressive loss of neuronal function.

Etiology. A number of etiological factors can be involved in the genesis of tinnitus. One of them is the hyperactivity of the auditory cortex and dorsal cochlear nucleus, damaged by oxidative stress. In addition, dysfunction between the central cortex

Table 1. Types of tinnitus

\begin{tabular}{|l|l|}
\hline Type of tinnitus & Characteristics \\
\hline Subjective & $\begin{array}{l}\text { The most common form of tinnitus. Subjective stimuli can only be heard by the affected person } \\
\text { and are usually caused by an exposure to an excessive noise. This type of tinnitus can appear and } \\
\text { disappear suddenly, and can last for 3-12 months. In some severe cases, it may never stop. }\end{array}$ \\
\hline Neurological & $\begin{array}{l}\text { It is usually caused by a disease, such as the Meniere's disease, which mainly affects the auditory } \\
\text { functions of the brain. }\end{array}$ \\
\hline Somatic & $\begin{array}{l}\text { Connected to the sensory system. This form is caused, aggravated or otherwise related to the } \\
\text { sensory system. }\end{array}$ \\
\hline Objective & $\begin{array}{l}\text { A rare form of tinnitus that can be caused by an involuntary muscle contractions or vascular } \\
\text { deformities. When the cause is treated, tinnitus usually stops completely. This is the only form } \\
\text { of tinnitus that can be heard by an outside observer, and the only type that has the potential for } \\
\text { permanent correction. }\end{array}$ \\
\hline
\end{tabular}

Table 2. Subtypes of tinnitus

\begin{tabular}{|l|l|}
\hline Subtypes of tinnitus & Characteristics \\
\hline Musical & $\begin{array}{l}\text { Also called musical hallucinations or auditory images, this type is less common. Simple } \\
\text { tones or layers of tones are collected to recreate a melody or a composition. Musical } \\
\text { tinnitus usually occurs in people who have had hearing loss and tinnitus for some time, } \\
\text { although people with normal hearing or hypersensitivity to sound may also have musical } \\
\text { hallucinations. }\end{array}$ \\
\hline Pulsating & $\begin{array}{l}\text { Rhythmic tinnitus that is in line with the heartbeat. It usually indicates a change in the blood } \\
\text { flow to the vessels near the ear or an increase in sensation of the blood flow to the ear. }\end{array}$ \\
\hline Low frequency & $\begin{array}{l}\text { The type that causes the most discomfort, as sufferers are not sure whether the sound is } \\
\text { produced internally or externally. Often the tones correspond to the two lowest octaves of a } \\
\text { piano and are described as buzzing, muttering or deep rumble. }\end{array}$ \\
\hline
\end{tabular}




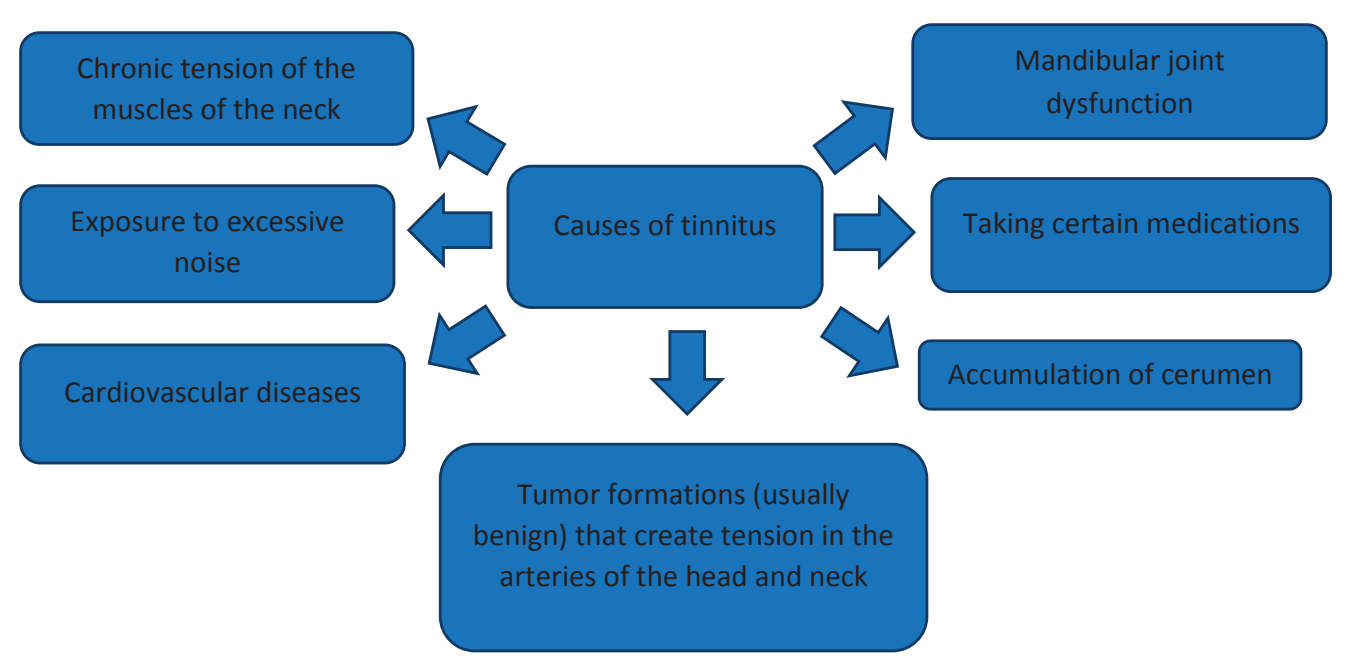

Figure 1. Main causes of tinnitus.

and inner ear is increasingly thought to be related to oxidative damage caused by free radicals [11]. One of the main causes of cell and tissue damage is the lipid peroxidation generated by reactive oxygen species. Oxidative stress and mitochondrial dysfunction are found in the early stages of severe neurodegenerative diseases [12].

The most common sensory disorder in the elderly is the hearing loss due to senile changes [13] where mitochondrial dysfunction as well as oxidative stress may play a role in the underlying pathology of the disease [14]. Evidence suggests that neuroanatomical changes in the brains of patients with dementia may involve the cortico-subcortical auditory and limbic networks. They may generate abnormal auditory perceptions [15]. The patient may have an expressionless face and a hunched posture. Impaired autonomic function can lead to a postural drop in blood pressure and hypersalivation.

To date, aging is the most significant risk factor for the neurodegenerative diseases. The number of older people has significantly increased and there is a corresponding rise in chronic degenerative diseases - such as Parkinson's disease. It has symptoms related to body balance and is becoming more common among the elderly population.

Cognitive etiology and consequences cannot be omitted. Studies have shown that central hearing dysfunction without severe peripheral hearing loss is associated with higher cases of cognitive decline and Alzheimer's disease [16], [17]. Both hearing loss and central hearing dysfunction are associated with a higher risk of developing dementia after 5-10 years [18]. In recent decades, structural and functional studies of the brain have demonstrated the link between tinnitus and various forms of cognitive dysfunction, especially impaired concentration and memory [19].

Studies have shown that chronic tinnitus is associated with anatomical brain abnormalities, including decreased cortical gray matter and decreased white matter integrity [20]. There is also a high prevalence of cognitive impairment reported among patients with tinnitus from a clinical point of view [21]. Tinnitus is an independent risk factor for subsequent $\mathrm{PD}$ and $\mathrm{AD}$. Although the causal relationship between tinnitus and AD / PD is unknown or even absent, the occurrence of tinnitus may be a possible risk factor for $\mathrm{AD} / \mathrm{PD}$.

In addition, whether the prevention and treatment of tinnitus may be helpful in reducing the incidence of $\mathrm{AD} / \mathrm{PD}$, a further research is required. However, there are some possible mechanisms to study the relationship between tinnitus and AD / PD. From a theoretical point of view, neurodegeneration is due to inflammation resulting from or prior to ischemia [22].

Scientists have concluded that there is an independent association in the incidence of $\mathrm{AD}$ with diabetes mellitus. In addition, the incidence of PD associated with a head injury, cerebrovascular disease and osteoarthritis have been established.

There is a wide variety of treatment options, but they differ in terms of effectiveness. More than 50 percent of those who experience tinnitus have hearing loss in the inner ear, which means a link between tinnitus and hearing loss is likely. Although wearing hearing aids helps to alleviate tinnitus (they amplify outside sounds, making the sounds inside more difficult to detect), they are not the only method: a 
careful diagnosis by a specialist is essential.

Pramipexole is an effective remedy for subjective tinnitus associated with presbyacusis in a dosing regimen used to treat Parkinson's disease. The drug does not change the threshold of hearing.

There are a number of neurological conditions that can be encountered in the otorhinolaryngological and dental practice. It is important for the dentist to have a broad knowledge of the underlying neurological conditions, as they may affect the provision of dental treatment. Our findings from the examinations executed are consistent with previous studies, which show that diabetes [23] and head injuries [24] can be risk factors for AD and neurodegeneration. On fig. 2 and 3, results from SERA-screening
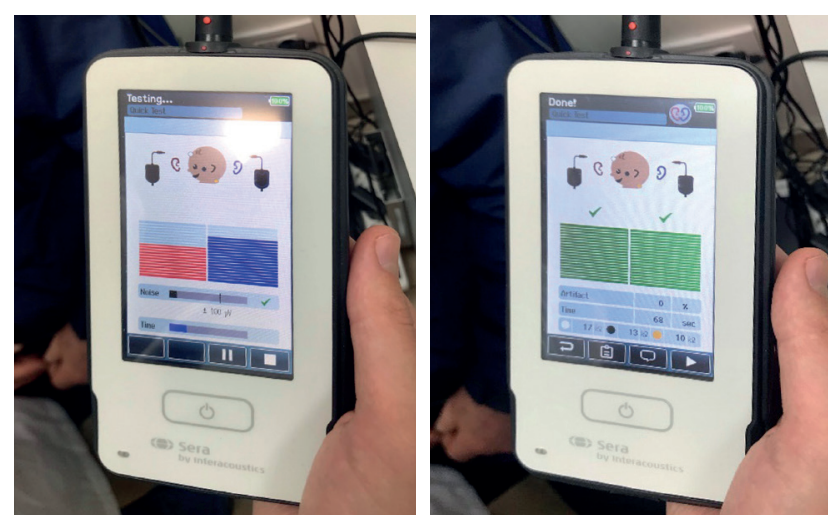

Fig. 2. SERA-examination and result
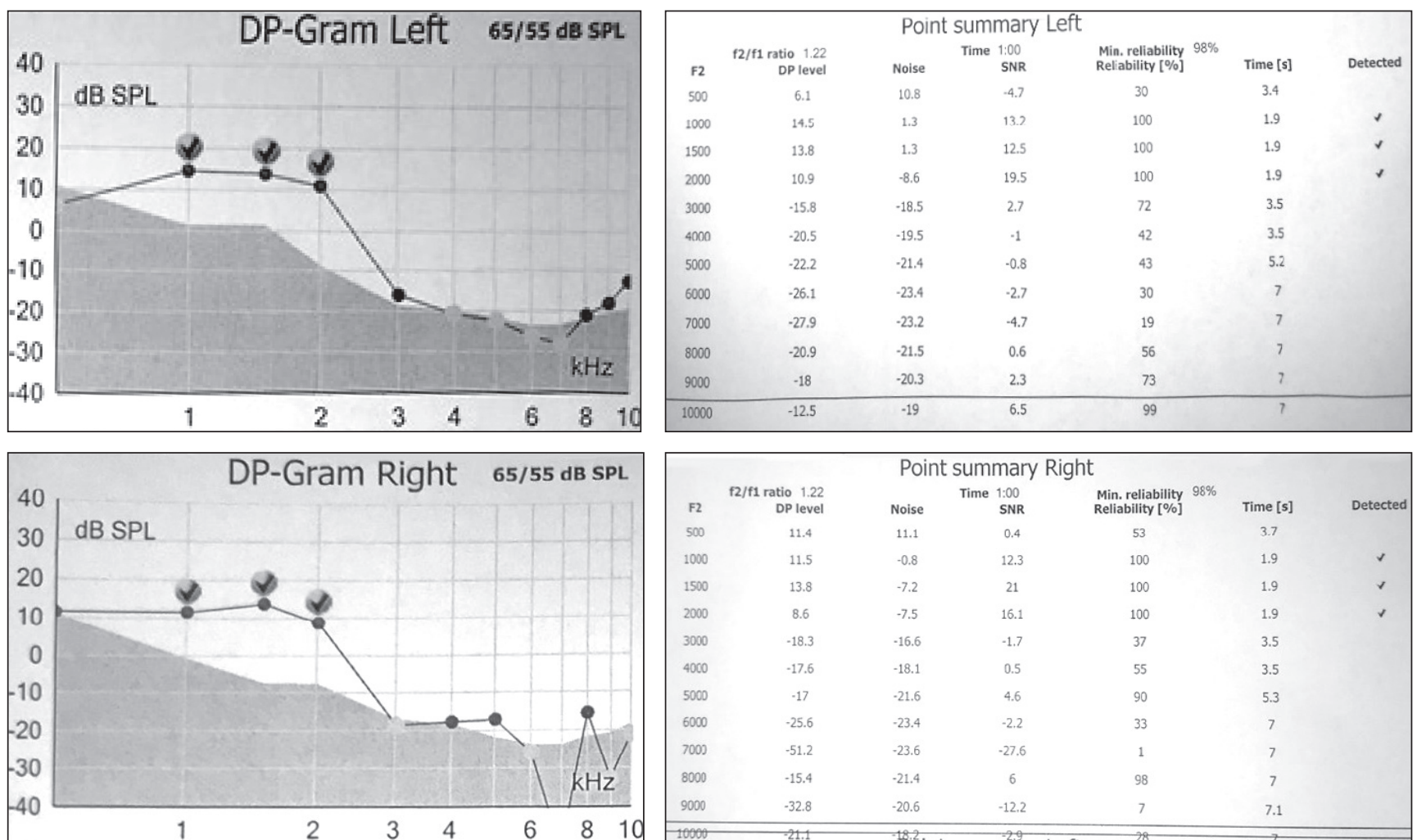

\begin{tabular}{|c|c|c|c|c|c|c|}
\hline \multicolumn{6}{|c|}{ Point summary Right } & \multirow[b]{2}{*}{ Detected } \\
\hline F2 & $\begin{array}{r}\text { f2/f1 ratio } 1.22 \\
\text { DP level }\end{array}$ & Noise & 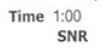 & $\begin{array}{l}\text { Min. reliability } 98 \% \\
\text { Reliability [\%] }\end{array}$ & Time [s] & \\
\hline 500 & 11.4 & 11.1 & 0.4 & 53 & 3.7 & \\
\hline 1000 & 11.5 & -0.8 & 12.3 & 100 & 1.9 & $\checkmark$ \\
\hline 1500 & 13.8 & -7.2 & 21 & 100 & 1.9 & $\checkmark$ \\
\hline 2000 & 8.6 & -7.5 & 16.1 & 100 & 1.9 & $\checkmark$ \\
\hline 3000 & -18.3 & -16.6 & -1.7 & 37 & 3.5 & \\
\hline 4000 & -17.6 & -18.1 & 0.5 & 55 & 3.5 & \\
\hline 5000 & -17 & -21.6 & 4.6 & 90 & 5.3 & \\
\hline 6000 & -25.6 & -23.4 & -2.2 & 33 & 7 & \\
\hline 7005 & -51.2 & -23.6 & -27.6 & 1 & 7 & \\
\hline 8000 & -15.4 & -21.4 & 6 & 98 & 7 & \\
\hline 9000 & -32.8 & -20.6 & -12.2 & 7 & 7.1 & \\
\hline
\end{tabular}

Fig. 3. DPOAE results - left and right ear and DPOAD-examination can be seen.

From the anamnesis, patient had pain and stiffness of the right shoulder and the waist. Patient reported changes in his hand-writing and gate. He was prescribed PK-Merz (Amantadine sulfate) and had responded well to the treatment - symptoms were alleviated. SPECT/CT (3.8 mCi 123-I StriaScan and low-dose CT of the head) was previously done in UMHAT "St. Marina" - Varna - Parkinson's disease was observed. No activity in left and right nucleus caudatus. No cortical regions accumulated the used radiopharmaceutical. No suspected pathologic regions of the head were observed with the low-dose CT.

\section{Conclusions}

Further research is needed to re-confirm the conclusions of our research and to investigate the pathophysiology of the relationship between tinnitus and the Alzheimer's disease and tinnitus and the Parkinson's disease in more depth. putamen regions, preserved activity in left and right 


\section{References}

1. Chari, Divya A., and Charles J. Limb. "Tinnitus.” Medical Clinics 102.6 (2018): 1081-1093.

2. DAVIS, ADRIAN C. "The prevalence of hearing impairment and reported hearing disability among adults in Great Britain." International journal of epidemiology 18.4 (1989): 911-917.

3. Demeester, K. et al. Prevalence of tinnitus and audiometric shape. B-ent 3(Suppl 7), 37-49 (2007).

4. Eggermont, Jos J. Hearing loss: Causes, prevention, and treatment. Academic Press, 2017

5. D. Baguley, D. McFerran, D. Hall. Tinnitus. Lancet, 382 (2013), pp. 1600-1607

6. Shulman, A., Goldstein, B. \& Strashun, A. M. Final common pathway for tinnitus: Theoretical and clinical implications of neuroanatomical substrates. Int. Tinnitus J. 15, 5-50 (2009)

7. A. Davis, E.A. Rafaie. Epidemiology of tinnitus. R.S. Tyler (Ed.), Tinnitus Handbook, Singular, San Diego, CA (2000)

8. M. Sereda, D.A. Hall, D.J. Bosnyak, M. Edmondson-Jones, L.E. Roberts, P. Adjamian, A.R. Palmer. Re-examining the relationship between audiometric profile and tinnitus pitch. Int. J. Audiol., 50 (2011), pp. 303-312

9. G. Barnea, J. Attias, S. Gold, A. Shahar. Tinnitus with normal hearing sensitivity: extended high-frequency audiometry and auditory-nerve brain-stem-evoked responses. Audiology, 29 (1990), pp. 36-45

10. Shulman, A., Goldstein, B. \& Strashun, A. M. Central nervous system neurodegeneration and tinnitus: A clinical experience. Part I: Diagnosis. Int. Tinnitus J. 13, 118-131 (2007)

11. Haase, G. M., Prasad, K. N., Cole, W. C., Baggett-Strehlau, J. M. \& Wyatt, S. E. Antioxidant micronutrient impact on hearing disorders: Concept, rationale, and evidence. Am. J. Otolaryngol. 32, 55-61. https://doi.org/10.1016/j.amjoto.2009.09.002 (2011)

12. Lin, Michael T., and M. Flint Beal. "Mitochondrial dysfunction and oxidative stress in neurodegenerative diseases." Nature 443.7113 (2006): 787-795

13. Gates, G. A. \& Mills, J. H. Presbycusis. Lancet 366, 1111-1120

14. Sugiyama, A. \& Sun, J. Immunochemical detection of lipid hydroperoxide- and aldehyde-modified proteins in diseases. Subcell. Biochem. 77, $115-125$

15. Mahoney, C. J. et al. Structural neuroanatomy of tinnitus and hyperacusis in semantic dementia. J. Neurol. Neurosurg. Psychiatry $82,1274-$ 1278

16. Gates, G. A., Anderson, M. L., Feeney, M. P., McCurry, S. M. \& Larson, E. B. Central auditory dysfunction in older persons with memory impairment or Alzheimer dementia. Arch. Otolaryngol. Head Neck Surg. 134, 771-777.(2008)

17. Gates, G. A., Anderson, M. L., McCurry, S. M., Feeney, M. P. \& Larson, E. B. Central auditory dysfunction as a harbinger of Alzheimer dementia. Arch. Otolaryngol. Head Neck Surg. 137, 390-395. (2011)

18. Albers, M. W. et al. At the interface of sensory and motor dysfunctions and Alzheimer's disease. Alzheimer's Dementia 11, 70-98. https://doi. org/10.1016/j.jalz.2014.04.514 (2015)

19. Jafari, Z., Kolb, B. E. \& Mohajerani, M. H. Age-related hearing loss and tinnitus, dementia risk, and auditory amplification outcomes. Ageing Res. Rev. 56, 100963. https://doi.org/10.1016/j.arr.2019.100963 (2019)

20. Adjamian, P., Hall, D. A., Palmer, A. R., Allan, T. W. \& Langers, D. R. M. Neuroanatomical abnormalities in chronic tinnitus in the human brain. Neurosci. Biobehav. Rev. 45, 119-133. https://doi.org/10.1016/j.neubiorev.2014.05.013 (2014)

21. Pierce, K. J. et al. Effects of severe bothersome tinnitus on cognitive function measured with standardized tests. J. Clin. Exp. Neuropsychol. 34, 126-134. https://doi.org/10.1080/13803395.2011.623120 (2012)

22. Shulman, A., Goldstein, B. \& Strashun, A. M. Central nervous system neurodegeneration and tinnitus: A clinical experience. Part I: Diagnosis. Int. Tinnitus J. 13, 118-131 (2007)

23. de Bruijn, R. F. A. G. \& Ikram, M. A. Cardiovascular risk factors and future risk of Alzheimer's disease. BMC Med. 12, 130. https://doi. org/10.1186/s12916-014-0130-5 (2014)

24. Gupta, R. \& Sen, N. Traumatic brain injury: A risk factor for neurodegenerative diseases. Rev. Neurosci. 27, $93-100$ (2016)

Reviewer of the article: Professor. Cem UZUN, MD, PhD

Balkan Society of Otorhinolaryngology Head-Neck Surgery (BSOHNS), General Secretary $43^{\text {rd }}$ National Congress of Turkish Society of Otorhinolaryngology Head-Neck Surgery, President Trakya Society of Otorhinolaryngology Head-Neck Surgery, President

\section{Correspondence to:}

Assoc. prof. Dr. Mario Milkov, MD, PhD

Department of Dental materials science and propaedeutics of prosthetic dental medicine, University medical and dental center, Faculty of Dental medicine, Medical University

"Prof. Dr. Paraskev Stoyanov" - Varna +359888897403

mario.milkov@gmail.com 